\title{
Harnessing Plasma Environments for Ammonia Catalysis: Mechanistic Insights from Experiments and Large-Scale Ab-initio Molecular Dynamics
}

\author{
Sharma S. R. K. C. Yamijala ${ }^{1 \dagger}$, Giorgio Nava ${ }^{2 \dagger}$, Zulfikhar A. Ali ${ }^{1}$, Davide Beretta ${ }^{3}$, Bryan M. Wong ${ }^{1,4^{*}}$, \\ Lorenzo Mangolini ${ }^{2,4 *}$ \\ ${ }^{1}$ Department of Chemical \& Environmental Engineering, University of California-Riverside, Riverside, \\ CA USA \\ ${ }^{2}$ Department of Mechanical Engineering, University of California-Riverside, Riverside, CA USA \\ ${ }^{3}$ Swiss Federal Laboratories for Materials Science and Technology, Dübendorf, Switzerland \\ ${ }^{4}$ Materials Science and Engineering Program, University of California-Riverside, Riverside, CA USA
}

\begin{abstract}
${ }^{\dagger}$ The first two authors equally contributed to this work
Corresponding authors: Bryan M. Wong (bryan.wong@ucr.edu) and Lorenzo Mangolini, (lmangolini@engr.ucr.edu)
\end{abstract}

By combining experimental measurements with ab initio molecular dynamics simulations, we provide the first microscopic description of the interaction between metal surfaces and a low-temperature nitrogen-hydrogen plasma. Our study focuses on the dissociation of hydrogen and nitrogen as the main activation route. We find that ammonia forms via an Eley-Rideal mechanism where atomic nitrogen abstracts hydrogen from the catalyst surface to form ammonia on an extremely short timescale (a few picoseconds). On copper, ammonia formation occurs via the interaction between plasma-produced atomic nitrogen and the H-terminated surface. On platinum, however, we find that surface saturation with $\mathrm{NH}$ groups is necessary for ammonia production to occur. Regardless of the metal surface, the reaction is limited by the mass transport of atomic nitrogen, consistent with the weak dependence on catalyst material that we observe and has been reported by several other groups. This study represents a significant step towards achieving a mechanistic, microscopic-scale understanding of catalytic processes activated in lowtemperature plasma environments. 


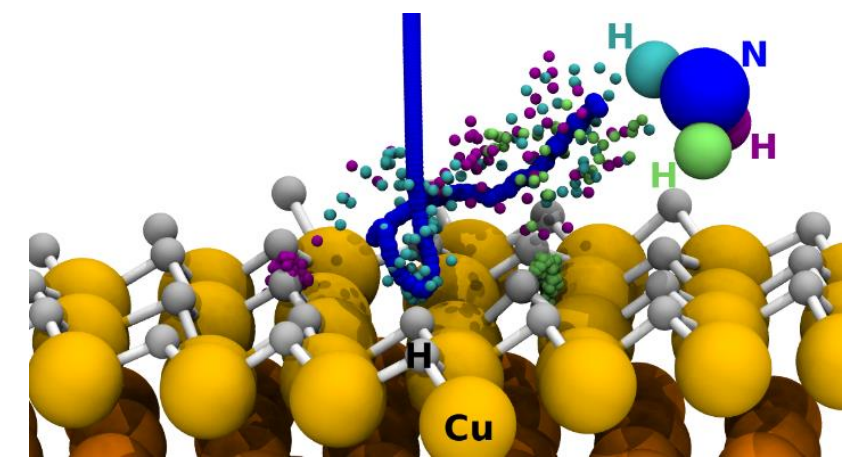


The use of low-temperature plasmas to drive heterogeneous chemical reactions continues to attract significant interest by both the plasma and catalysis communities. ${ }^{1-14}$ Since these processes are electrically driven, they are compatible with a decentralized, renewable energy-driven chemical manufacturing infrastructure. To this end, many chemical reactions are currently being investigated by the plasma community, such as methane reforming, ${ }^{1-3} \mathrm{CO}_{2}$ hydrogenation, ${ }^{10-11}$ and others. ${ }^{7,}{ }^{15}$ Among these reactions, the case of plasma-driven nitrogen fixation is particularly interesting because its thermal counterpart - the Haber-Bosch process, ${ }^{16-19}$ which is the standard for the industrial production of ammonia - is particularly energy-intensive. Reasonably high degrees of nitrogen conversion to ammonia have been reported for a broad range of reactor designs, varying from atmospheric pressure dielectric barrier discharges (DBDs) in contact with a catalyst bed ${ }^{6,20}$ to low-pressure plasmas driven by radio-frequency excitation. ${ }^{21-22}$ No external heating is required in any of these cases, and good degrees of nitrogen fixation, even with the catalyst at nominally room temperature, are observed. The exact mechanism leading to the reasonably efficient fixation of nitrogen in these systems is still under intense debate. Mehta et al. ${ }^{6}$ attributed the reasonably high yield of ammonia in a dielectric barrier discharge to the plasma-induced vibrational excitation of nitrogen, effectively lowering the barrier for dissociation upon adsorption onto the catalyst surface. This results in an increased activity for metals such as Co, i.e., catalysts that interact more weakly with nitrogen (with a more positive nitrogen adsorption energy) compared to those optimized for the Haber-Bosch process such as Ru. In addition, Mehta et al. ${ }^{6}$ predicted a strong dependence of reaction turnover frequency over the catalyst material, varying by orders of magnitude. Iwamoto et al. ${ }^{23}$ compared the activity of different materials by placing metallic wool within a tubular plasma reactor operated at atmospheric pressure, making sure that the area exposed to the plasma is constant between the different materials. They found a very similar ammonia yield (well within a factor of two) for metals such as $\mathrm{Pt}, \mathrm{Au}, \mathrm{Ag}, \mathrm{Cu}, \mathrm{Pd}$, and $\mathrm{Fe}$. In the thermally activated case, the reactivity of these metals would differ by several orders of magnitude. Iwamoto et al. ${ }^{23}$ attempted to gain a microscopic understanding of this behavior by performing atomistic DFT calculations. They found a reasonable correlation between ammonia yield and the stability of surface $\mathrm{M}_{3} \mathrm{~N}$ groups, where $\mathrm{M}$ is the metal. Shah et al. ${ }^{21}$ impinged a low pressure $\left(0.26\right.$ Torr) radio-frequency $\mathrm{N}_{2^{-}}$ $\mathrm{H}_{2}$ plasma onto metallic meshes of $\mathrm{Cu}, \mathrm{Pd}, \mathrm{Ag}$, and $\mathrm{Au}$, and obtained effectively the same ammonia yield, suggesting a weak dependence on catalyst material. The authors performed a detailed microkinetic analysis for the case of $\mathrm{Fe}$, supporting the hypothesis that nitrogen dissociation is the main activation mechanism in the low pressure regime. Van Helden et al. ${ }^{24}$ generated a $\mathrm{N}_{2}-\mathrm{H}_{2}$ plasma via a high-temperature plasma torch, followed by expansion of the ionized gas into a low-pressure chamber. The authors used emission spectroscopy to determine the concentration of ammonia in the gas-phase and found the same value after the stainless steel reactor walls were coated with a silicon nitride layer. The authors concluded that ammonia was produced at the reactor walls via recombination of plasma-produced radicals, consistent with a lack of 
material dependence. Finally, Hong et al. ${ }^{8}$ demonstrated a reasonably high yield of ammonia even when an atmospheric pressure low-temperature plasma was in contact with a diamond-like carbon surface, suggesting a reaction pathway that is drastically different from that of a thermally-activated process. Overall, the scientific community agrees that excitation of nitrogen in the gas-phase is critical, with the accepted dominant mechanism being vibrational excitation for atmospheric pressure processes because of the reduced electric field strength and lower electron temperature. ${ }^{25}$ Nitrogen dissociation is generally considered to be prevalent at lower pressure. Both Eley-Rideal and Langmuir-Hinshelwood mechanisms are generally invoked to explain the formation of ammonia in plasma-assisted processes. ${ }^{21,26}$

Still, significant gaps in knowledge exist with respect to the interaction between plasma-produced species and catalyst surfaces. While reactor-level characterization of ammonia yield, in combination with micro-kinetic models, can be useful from the point of view of process design and optimization, this strategy is limited as it cannot provide a mechanistic, microscopic understanding of surface reaction pathways. Common atomistic models such as DFT are limited to static, zero kelvin configurations, and therefore cannot predict chemical configurations in a dynamic environment. In this manuscript, we address this knowledge gap by combining a reactor-level characterization of a low-pressure RF plasma operating in a hydrogen-nitrogen mixture complemented with large-scale Born-Oppenheimer Molecular Dynamics (BOMD) simulations. We focus on a low-pressure, RF-sustained plasma since these conditions are conducive to a spatially and temporally uniform plasma, as opposed to dielectric barrier discharges, which are composed of transient and highly localized plasma filaments, complicating the plasma-surface interaction and the interpretation of the measurements. To complement our experimental measurements, we utilize BOMD calculations since this technique provides a direct, dynamical, and unbiased approach for predicting reaction mechanisms on surfaces. ${ }^{27-29}$ Since BOMD includes both dynamic and steric effects, it naturally gives unbiased results compared to static electronic structure methods such as conventional density functional theory (DFT). Also, since the potential energy surfaces (which dictate the forces on the nuclei) are computed in an ab initio manner during a BOMD simulation, it is superior to classical molecular dynamics and empirical force-field techniques. Its capability of handling radicals such as atomic nitrogen and hydrogen (via spin-polarization quantum mechanical effects) makes it essential for investigating plasma-produced ammonia processes in low-pressure reactors, where the dissociation of $\mathrm{N}_{2}$ and $\mathrm{H}_{2}$ is the dominant excitation mechanism.

The measurements are performed on a RF-driven plasma operating in a 1:3 $\mathrm{N}_{2}: \mathrm{H}_{2}$ mixture at a pressure of 1 Torr. The system is comprised of a quartz cylindrical plasma reactor with three ring electrodes, two of which are grounded while the center electrode is connected to a radiofrequency (RF) power source. The tube diameter is 1", with each electrode being 1" wide. The plasma is ignited in a mixture of nitrogen 
flowing at 20 standard cubic centimeters per minute $(\mathrm{sccm})$ and hydrogen flowing at $60 \mathrm{sccm}$. A schematic and a photograph of the reactor are shown in Figure 1a. A Faraday cage is used to prevent interference with the surrounding electronics. Part of the exhaust stream is sampled through a $50 \mu \mathrm{m}$ orifice expanded into a low-pressure chamber onto which a residual gas analyzer (RGA) is mounted. The transmission coefficient of the orifice is dependent on the atomic mass unit (amu) of the sampled species. We have carefully calibrated the response by flowing known amounts of $\mathrm{N}_{2}, \mathrm{H}_{2}$, and $\mathrm{NH}_{3}$ though the reactor. Details of the calibration procedure are given in the Supplementary Information document (see Figure S1).

The catalyst consists of an aluminum foil substrate (1"×6") onto which $100 \mathrm{~nm}$ metallic films are applied via DC magnetron sputtering. The foil is then rolled into a cylinder to line a portion of the inside wall of the reactor. This approach ensures that the catalyst-active area is identical when different materials are compared. It is important to note that the position of the catalyst in the tube reactor is critical. We observed a negligible ammonia yield when the catalyst is placed in the initial section of the reactor, i.e., under the grounded electrode placed immediately upstream of the RF biased electrode. This low yield is an expected result since the ammonia produced at the entrance of the reactor rapidly dissociates as it travels through the plasma. We have further verified this hypothesis using a control experiment, where we added a known amount of ammonia to the gas stream without placing any catalyst (metal foil) into the reactor. For this experimental setup, plasma ignition results in the decomposition of $>90 \%$ of the ammonia supplied at the inlet, strongly supporting our hypothesis. To ensure higher ammonia yields, in all our experiments, we placed the catalyst under the downstream grounded electrode, i.e., closer to the exit of the plasma volume. The residence time through the plasma volume at 1 Torr, based on the flow velocity, is $30 \mathrm{msec}$.

The yield of ammonia is calculated by monitoring the partial pressures of nitrogen at amu 28 and ammonia at amu 17. The degree of molecular fragmentation induced by the electron gun of the RGA is accounted for in the calculations. In this manuscript, we report the nitrogen fixation efficiency $\eta$, i.e., the atomic fraction of nitrogen carried by ammonia at the reactor exhaust, defined as:

$$
\eta=\frac{P_{N H_{3}}}{2 \cdot P_{N_{2}}+P_{N H_{3}}}
$$

where $\mathrm{P}_{\mathrm{NH} 3}$ and $\mathrm{P}_{\mathrm{N} 2}$ are the partial pressures of ammonia and nitrogen downstream of the plasma volume, respectively. In Figure 1b, we show the measured fixation efficiency at a power of $150 \mathrm{~W}$ for different metals. In the absence of any catalyst (labeled as "No Catalyst" in Figure 1b) we reproducibly observed a yield of roughly $0.5 \%$, which can be attributed to ammonia production at the quartz surface. The $\mathrm{Al}$ and $\mathrm{Ti}$ surfaces did not show any increase in the yield compared to the "no catalyst" case. We strongly suspect that this inactivity is due to their native oxide layer, which makes their catalytic activity comparable to that of quartz. Among the other metals, Mo yielded roughly $0.75 \%$ of ammonia, while $\mathrm{Ag}, \mathrm{Cu}, \mathrm{Pd}, \mathrm{Co}$, and $\mathrm{Ni}$ 
behaved very similarly, yielding $\sim 1.25 \%$ of ammonia. Pt and Fe produced around $1.6 \%$ of ammonia. As a whole we observed a weak dependence on catalyst material, consistent with other reports in the literature. Also, we found a roughly linear dependence of the ammonia yield on the RF input power (as shown in Figure S2). It is important to note that using a too high input power (above 150W) led to sputtering of the metal and contamination of the reactor tube. We, therefore, do not report those results. The error bars were determined by repeating the measurement with the $\mathrm{Al}$ foil five times and picking the maximum spread among those as the measured error.
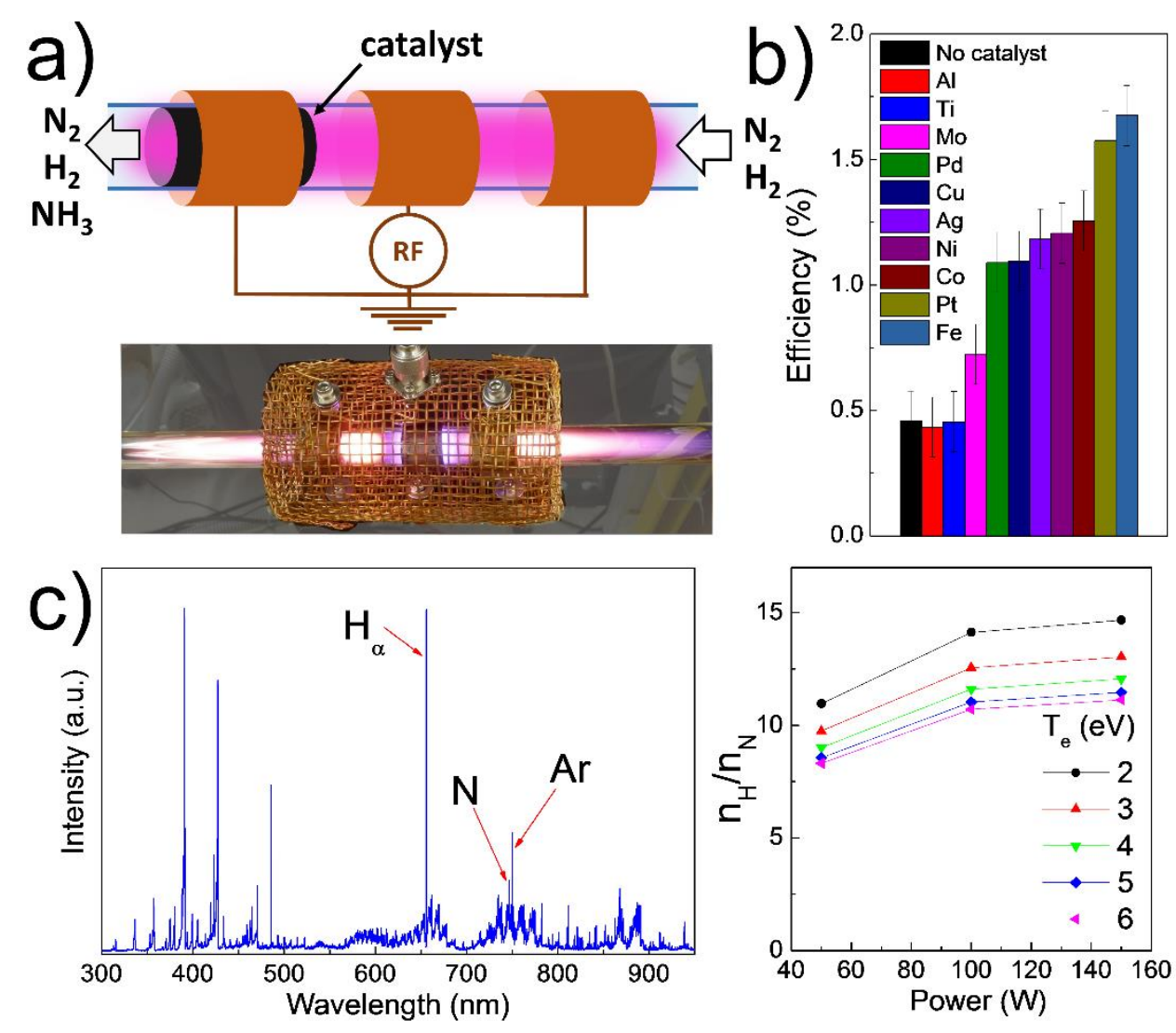

Figure 1. (a) Schematic and photograph of the flow-through RF-driven plasma reactor for the fixation of $\mathrm{N}_{2}$ and production of ammonia. (b) Histogram comparing the nitrogen fixation efficiency, as defined in equation (1), for a power input of $150 \mathrm{~W}$ and a pressure of 1 Torr, for different catalytic surfaces. (c) Example of an optical emission spectrum observed for a nitrogen-hydrogen mixture, with the small addition $(5 \%)$ of argon as an actinomer. For clarity, the amplitude of the $\mathrm{H}_{\alpha}$ peak has been reduced by a factor of 5 . The right panel also shows the ratio between atomic hydrogen and nitrogen densities, as obtained by the OES data, following the procedure outline in the text.

We performed optical emission spectroscopy (OES) to estimate the density of atomic species in the plasma since atomic nitrogen is the main activated species in the low-pressure plasma regime. A small flow 
of argon has been added to the nitrogen-hydrogen mixture as an actinomer, ${ }^{30}$ and the density of atomic nitrogen and atomic hydrogen have been obtained by the emission line intensity ratios using the procedure outlined by Tatarova et al. ${ }^{31}$ An example of the spectrum used for the calculation is shown in Figure 1c. We have used the following emission lines for our calculation: the $\mathrm{H}_{\alpha}$ hydrogen line at $656.2 \mathrm{~nm}$ (transition $3 \mathrm{~d}^{2} \mathrm{D}_{\mathrm{j}}$ to $2 \mathrm{p}^{2} \mathrm{P}_{0}$ ), the nitrogen line at $744.2 \mathrm{~nm}$ (transition $3 \mathrm{p}^{4} \mathrm{~S}^{0}$ to $\left.3 \mathrm{~s}^{4} \mathrm{P}\right)$, and the $750.4 \mathrm{~nm}$ line for $\operatorname{Ar}\left(2 \mathrm{p}^{1}\right.$ to $1 \mathrm{~s}^{2}$ transition). The following relation was used to calculate the densities of atomic nitrogen and hydrogen, respectively:

$$
\begin{aligned}
& \frac{n_{N}}{n_{A r}}=\frac{I(744)}{I(750)} \cdot \frac{744}{750} \cdot\left(\frac{X_{A r}}{X_{N}}\right) \cdot \frac{A_{750} /\left(\sum A_{j, A r}+K_{M}^{A r} \cdot n_{M}\right)}{A_{744} /\left(\sum A_{j, N}+K_{M}^{N} \cdot n_{M}\right)}, \\
& \frac{n_{H}}{n_{A r}}=\frac{I(656)}{I(750)} \cdot \frac{656}{750} \cdot\left(\frac{X_{A r}}{X_{H}}\right) \cdot \frac{A_{750} /\left(\sum A_{j, A r}+K_{M}^{A r} \cdot n_{M}\right)}{A_{656} / \sum A_{j, H}},
\end{aligned}
$$

where $n_{N}$ and $n_{H}$ are the densities of atomic nitrogen and hydrogen, and $n_{A r}$ is the density of argon as determined by ideal gas law. I(744), I(750), and I(656) are the emission intensities from the atomic species at those wavelengths corresponding to nitrogen, argon, and hydrogen, respectively. $\mathrm{A}_{744}, \mathrm{~A}_{750}$, and $\mathrm{A}_{656}$ are the transition probabilities for the corresponding lines (numerical values are provided in the Supplementary Information). $\mathrm{A}_{\mathrm{j}, \mathrm{Ar}}, \mathrm{A}_{\mathrm{j}, \mathrm{N}}$, and $\mathrm{A}_{\mathrm{j}, \mathrm{H}}$ are the transition probabilities for all other optically allowed transitions from the same excited states for the corresponding atomic species, which need to be accounted for to obtain the correct branching ratio. The transition probabilities are obtained from the NIST atomic spectra database. ${ }^{32} \mathrm{~K}^{\mathrm{Ar}}{ }_{\mathrm{M}}$ and $\mathrm{K}^{\mathrm{N}}{ }_{\mathrm{M}}$ are quenching rates that account for the non-radiative relaxation of the excited state of Ar and $\mathrm{N}$ respectively due to collision with partner $\mathrm{M}$, with $\mathrm{M}=\mathrm{Ar}, \mathrm{N}_{2}, \mathrm{H}_{2}$. We use the same values utilized by Tatarova et al. $^{31}$, with the numerical values provided in the Supplementary Information. $\mathrm{n}_{\mathrm{M}}$ is the density of the collision partner, as determined by ideal gas law. $\mathrm{X}_{\mathrm{Ar}}, \mathrm{X}_{\mathrm{N}}$, and $\mathrm{X}_{\mathrm{H}}$ are the electron impactinduced excitation rates to the selected excited states of argon, nitrogen, and hydrogen. The excitation rates are obtained by averaging the electron-induced excitation cross sections ${ }^{33}$ over the electron energy distribution function (EEDF). While Tatarova et al. ${ }^{31}$ assumed a Maxwell-Boltzmann EEDF, here we use Bolsig,$+{ }^{34,35}$ a commonly utilized freeware software for the solution of the Boltzmann transport equation, to obtain the EEDF. It is well-known that the EEDF strongly depends on the electron temperature $\mathrm{T}_{\mathrm{e}}$. Since $\mathrm{T}_{\mathrm{e}}$ is an unknown variable, we calculated the EEDF over a wide range of $\mathrm{T}_{\mathrm{e}}$ (between 2 and $6 \mathrm{eV}$ ), and the corresponding EEDFs are shown in Figure S3. The atomic nitrogen and hydrogen densities obtained following this procedure are shown in Figure S4 as a function of input RF power and electron temperature. For a power of $150 \mathrm{~W}$ and $T_{e}=6 \mathrm{eV}$, we find that the atomic hydrogen density $\mathrm{n}_{\mathrm{H}}$ is $\sim 4 \times 10^{14} \mathrm{~cm}^{-3}$, and the atomic nitrogen density $\mathrm{n}_{\mathrm{N}}$ is roughly one order of magnitude smaller, $\sim 4 \times 10^{13} \mathrm{~cm}^{-3}$. In Figure 1c, we also show the ratio between atomic hydrogen and nitrogen densities. For all conditions assumed here, the atomic 
hydrogen density exceeds that of atomic nitrogen by at least a factor of 5 , with a weak dependence on the electron temperature. These density values are consistent with those from other groups. ${ }^{36-39}$ Tatarova et al. $^{31}$ report a nitrogen degree of dissociation as high as $4 \%$, although they investigate a more energy-dense, microwave sustained plasma.

Overall, our measurements confirm the weak dependence of ammonia yield on the catalyst material. Surprisingly, we observed effectively the same yield on Pt and Fe, despite Pt being an inactive catalyst under thermally-activated conditions because of its more positive nitrogen adsorption energy of 0.6 $\mathrm{eV}^{6}$ (i.e., nitrogen does not absorb on Pt as strongly as on Fe). Similarly, we observed a good yield even for $\mathrm{Cu}(1.1 \%$ compared to $1.6 \%$ for Pt at $150 \mathrm{~W}$ RF power), despite DFT calculations predicting a very high nitrogen adsorption energy of $3.4 \mathrm{eV}$ for this metal ${ }^{40}$ (i.e., nitrogen does not absorb on $\mathrm{Cu}$ ). In addition, the OES characterization suggests that for all the conditions under consideration here, the atomic hydrogen density exceeds that of atomic nitrogen (see Figure 1c). Therefore the flux of atomic hydrogen to the catalyst surface greatly exceeds that of atomic nitrogen, which implies a higher probability of formation of a hydrogen-terminated surface under plasma exposure. As such, it is necessary to investigate the interaction between the plasma-produced species and the hydrogen-terminated surfaces to advance our understanding of plasma-driven heterogeneous catalysis. To this end, we performed large-scale BOMD simulations of atomic nitrogen impinging onto hydrogen-terminated catalyst surfaces. Given the fact that these simulations are computationally demanding, we focused on two metals, namely, $\mathrm{Cu}$ and Pt. Both of these metals adsorb nitrogen more weakly compared to the optimal choice for a thermally driven process (they are on the right branch of the well-known volcano plot for ammonia synthesis) and show good ammonia yield for a plasmadriven reaction, consistent with other reports in the literature. They also have greatly different nitrogen adsorption energies, with the case of $\mathrm{Cu}(+3.4 \mathrm{eV})^{40}$ being significantly more positive than that of $\mathrm{Pt}(+0.6$ $e V)^{6}$. Our own calculations of the nitrogen binding energies for $\mathrm{Cu}(111)$ and $\mathrm{Pt}(111)$ surfaces find values of $-3.681 \mathrm{eV}$ and $-5.115 \mathrm{eV}$, respectively (see table $\mathrm{S} 1$ ), consistent with the more positive adsorption energy for copper. It is, therefore, interesting to investigate the microscopic behavior of these metals, given their similar yields of ammonia under plasma exposure.

We performed spin-polarized, ab initio Born-Oppenheimer molecular dynamics (BOMD) simulations with three-layer $6 \times 6-\mathrm{Cu}(111)$ and $\mathrm{Pt}(111)$ surfaces, where the atomic positions of the bottom layer (36 metal atoms) are fixed. We passivated all the metal atoms in the top-layer with either hydrogen or nitrogen. First, we optimized these passivated structures using DFT and conducted NVT simulations to check their stability at room temperature. We used the Nosé-Hoover thermostat of the chain length three to maintain the temperature at $300 \mathrm{~K}$ and subsequently simulated the impingement of nitrogen atoms onto $\mathrm{H}$ terminated surfaces. The initial positions and velocities for all the NVE simulations were obtained from the 
NVT simulations, and a 0.5 fs time step was used to integrate the equations of motion. For the impinging nitrogen atom, the initial velocity was set such that it has $0.1 \mathrm{eV}$ kinetic energy towards the surface (we tried with other incidence energies in the range of $0.03-0.3 \mathrm{eV}$, and all of them gave similar results (see Figure S10)), and its initial position was kept $5 \AA$ above the passivated surface. To avoid any spurious interactions between the periodic images, $15 \AA$ of vacuum space was used. On the hydrogen-terminated surfaces, we impinged an $\mathrm{N}$ atom at 9 different angles, from 0 to 80 degrees at 10 -degree intervals. The results for the case of $\mathrm{Cu}$ are presented first in Figure 2. Figure 2a shows the hydrogen-terminated $\mathrm{Cu}$ surface at $0 \mathrm{~K}$ and after equilibration at $300 \mathrm{~K}$, confirming the stability of the hydrogen termination at room temperature. A similar simulation with a nitrogen-terminated $\mathrm{Cu}$ surface at $300 \mathrm{~K}$ (Figure 2b) resulted in the facile desorption of the nitrogen molecules. This observation is consistent with the high adsorption energy of nitrogen on copper. In Figure 2c, we show the NVE simulation results of the atomic nitrogen 

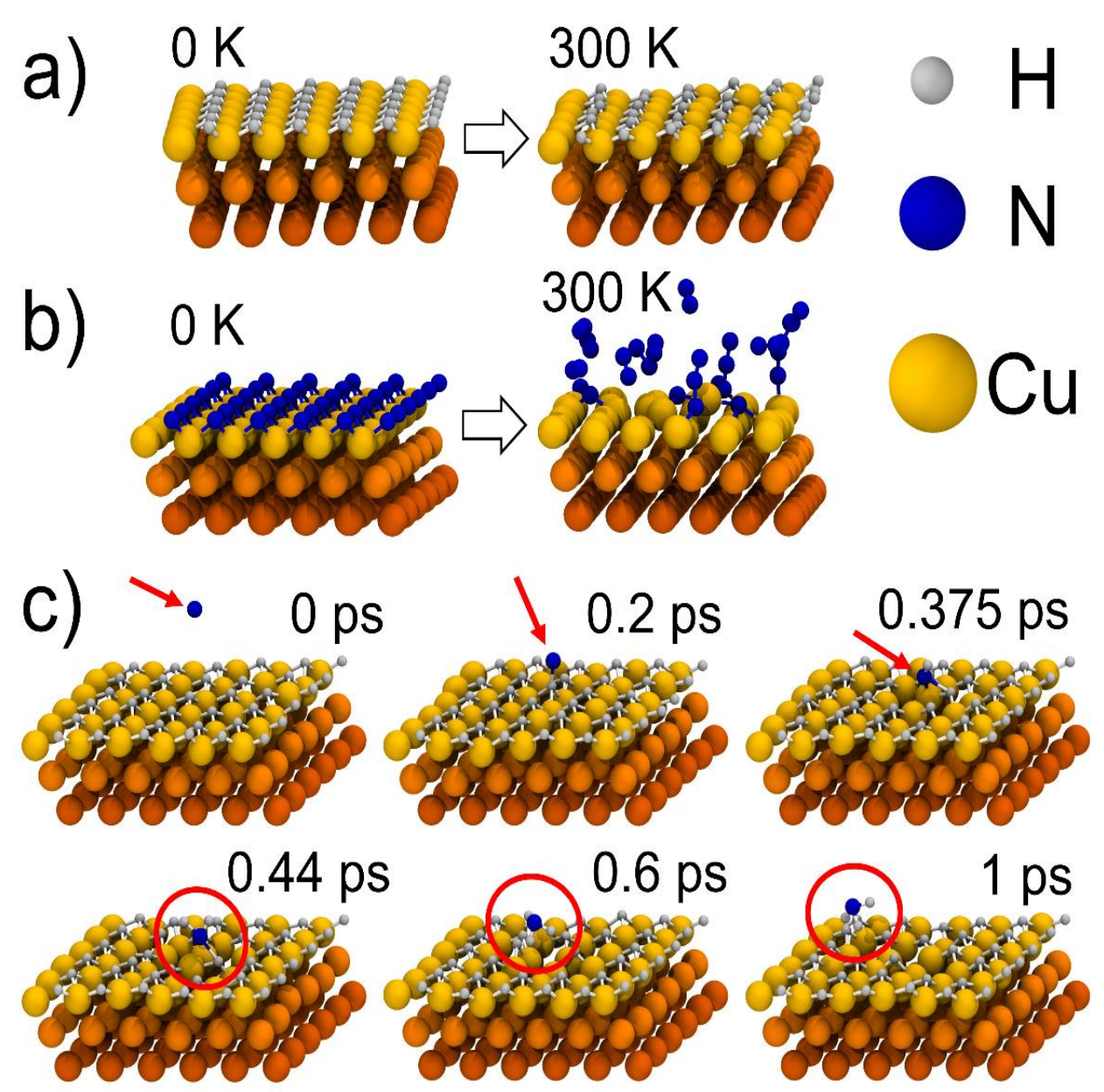

Figure 2. Optimized geometry (left) and the geometry after a 1 ps long NVT simulation (right) of an (a) H-terminated and (b) N-terminated $\mathrm{Cu}(111)$ surface. At room temperature, only hydrogen termination was found to be stable whereas nitrogen termination led to the desorption of $\mathrm{N}_{2}$ molecules from the $\mathrm{Cu}$ surface. (c) Atomic geometries at various time steps during an NVE simulation of an atomic nitrogen impinging onto a $\mathrm{H}$-terminated $\mathrm{Cu}(111)$ surface at normal incidence, with a kinetic energy of $0.1 \mathrm{eV}$. In the initial frame of this NVE simulation, the $\mathrm{N}$ atom was placed at $5 \AA$ above the surface. The outcomes for eight other angles of incidence are given in Figure S5. For all conditions, either $\mathrm{NH}_{3}$ or $\mathrm{NH}_{2}$ is the reaction product. The three layers of the copper surface are shown in different shades of orange/yellow color.

impinging onto an $\mathrm{H}$-terminated $\mathrm{Cu}$ surface with six consecutive snapshots spanning an interval of $1 \mathrm{ps}$. The corresponding energy profiles (see Figure S7) and movies are available in the Supplementary Information. These NVE results clearly suggest that the incoming nitrogen atom readily abstracts hydrogen atoms from the surface and rapidly desorbs as ammonia (within $1 \mathrm{ps).} \mathrm{The} \mathrm{time-dependent} \mathrm{bond} \mathrm{distance}$ and angles of the formed $\mathrm{NH}_{3}$ molecules are shown in Figure $\mathrm{S} 11$. The outcome of NVE simulations performed at different angles of incidence is shown in Figure S5. For all the incident angles, we consistently 
observed the formation and desorption of $\mathrm{NH}_{3}$ or $\mathrm{NH}_{2}$ (accompanied by the desorption of $\mathrm{H}_{2}$ in a few cases) from the $\mathrm{Cu}$ surface.

In stark contrast, the BOMD simulation results on the Pt surface are drastically different from the $\mathrm{Cu}$ surface, as summarized in Figure 3. Figure $3 \mathrm{a}$ and $3 \mathrm{~b}$ show the results of NVT simulations on Pt for determining the stability of $\mathrm{H}$-termination and $\mathrm{N}$-termination, respectively. As expected, hydrogen is stable at room temperature on Pt. Nitrogen is also stable on $\mathrm{Pt}$ at room temperature, showing a very different behavior compared to $\mathrm{Cu}$ (Figure $2 \mathrm{~b}$ ), which instead showed rapid desorption of nitrogen molecules from the surface. This is consistent with the less positive adsorption energy of nitrogen on $\mathrm{Pt}$ compared to $\mathrm{Cu}$. Moreover, the NVE simulations for Pt (Figure 3c) have a very different outcome compared to the case for $\mathrm{Cu}$ (Figure 2c). Impinging atomic nitrogen does not lead to the formation of $\mathrm{NH}_{2} \mathrm{Or} \mathrm{NH}_{3}$ molecules; rather, it sticks onto the Pt surface without formation of an $\mathrm{N}-\mathrm{H}$ bond, at least within the time window considered

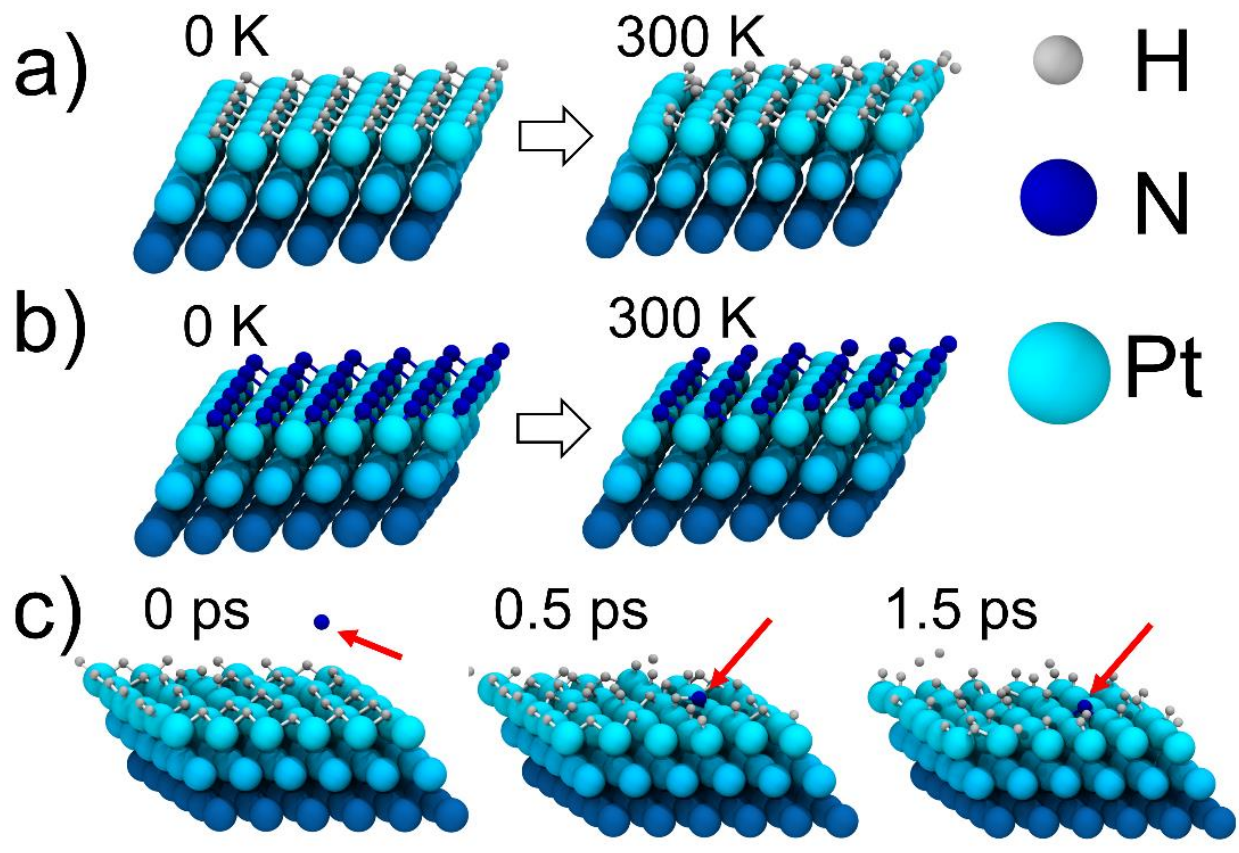

Figure 3. Optimized geometry (left) and the geometry after a 2 ps long NVT simulation (right) of an (a) H-terminated and (b) N-terminated Pt(111) surface. At room temperature, both hydrogen and nitrogen terminations were found to be stable for a Pt surface. (c) Atomic geometries at various time steps during an NVE simulation of an atomic nitrogen impinging onto a $\mathrm{H}$-terminated $\operatorname{Pt}(111)$ surface at a $20^{\circ}$ angle to the surface normal, with a kinetic energy of $0.1 \mathrm{eV}$. In the initial frame of this NVE simulation, the $\mathrm{N}$ atom was placed at 5 $\AA$ above the surface. The outcomes for eight other angles of incidence are given in Figure S6. For most of the cases, we observe a strong adsorption of the nitrogen atom onto the Pt surface, without any immediate $N$ - $H$ bond formation. The three layers of the platinum surface are shown in different shades of cyan/blue color. 
here ( $<2$ ps). The corresponding energy profiles (Figure S8) and movies are available in the Supplementary Information. The outcomes of the same NVE simulation performed for different nitrogen impinging angles are shown in Figure S6. For the majority of cases, we observe strong adsorption of nitrogen onto the Pt surface without immediate formation of an N-H bond. However, in one case, we observed the formation of an N-H surface group, and in another case, the formation of $\mathrm{NH}_{3}$. So while these calculations do not rule out the formation of ammonia as a result of $\mathrm{N}$-impingement onto $\mathrm{H}$-terminated $\mathrm{Pt}$, they do suggest that the likelihood of such reaction pathway is considerably smaller for $\mathrm{Pt}$ compared to $\mathrm{Cu}$. This lower probability is a consequence of the stronger interaction of atomic nitrogen with $\mathrm{Pt}$ compared to $\mathrm{Cu}$ (see Figures $2 \mathrm{~b}$ and $3 \mathrm{~b}$, and table $\mathrm{S} 1)$.

The above results suggest that the same microscopic mechanism, i.e., a direct abstraction of hydrogen atoms (from an $\mathrm{H}$-terminated catalyst surface) by the impinging nitrogen atom, cannot explain the similar yields of ammonia for hydrogen-terminated $\mathrm{Cu}$ and $\mathrm{Pt}$ surfaces. To solve this discrepancy, we first assumed that the continuous impingement of atomic hydrogen and nitrogen onto the Pt surface ultimately leads to the formation of an NH-termination. This assumption is justified by the significantly larger density of atomic hydrogen compared to atomic nitrogen (Figure 1c), with the corresponding thermal flux of atomic hydrogen exceeding that of atomic nitrogen by an even larger factor. Next, we performed BOMD calculations on this NH-terminated Pt, as shown in Figure 4. The NVT simulations in Figure 4a suggest that the $\mathrm{NH}$ termination is not entirely stable at room temperature, with some $\mathrm{NH}$ and $\mathrm{NH}_{2}$ fragments desorbing from the surface. Most importantly, we observe that the direct formation of $\mathrm{NH}_{3}$ from the $\mathrm{NH}$-terminated $\mathrm{Pt}$ is possible under exposure to either atomic hydrogen or nitrogen within the computational time scales accessible to the BOMD technique. In Figure 4b, we show results from the case of atomic hydrogen impinging onto the NH-terminated surface. The formation and desorption of $\mathrm{NH}_{3}$ are clearly observed (see Figures S12 and S13 for the time-dependent bond-distance and angles of the formed $\mathrm{NH}_{3}$ molecules). In Figure 4c, we show the case of atomic nitrogen impinging onto the NH-terminated Pt surface. Within $1 \mathrm{ps}$, we observe the formation and desorption of ammonia, similar to what is observed for the case of H-terminated $\mathrm{Cu}$. The corresponding energy profiles (Figure S9) and movies are available in the Supplementary Information.

Overall, these results suggest that there are important differences in the mechanism of ammonia formation for the case of $\mathrm{Pt}$ and $\mathrm{Cu}$. For $\mathrm{Cu}$, nitrogen does not adsorb onto the surface, and ammonia is formed via an Eley-Rideal mechanism leading to the direct abstraction of surface-bound hydrogen by the 


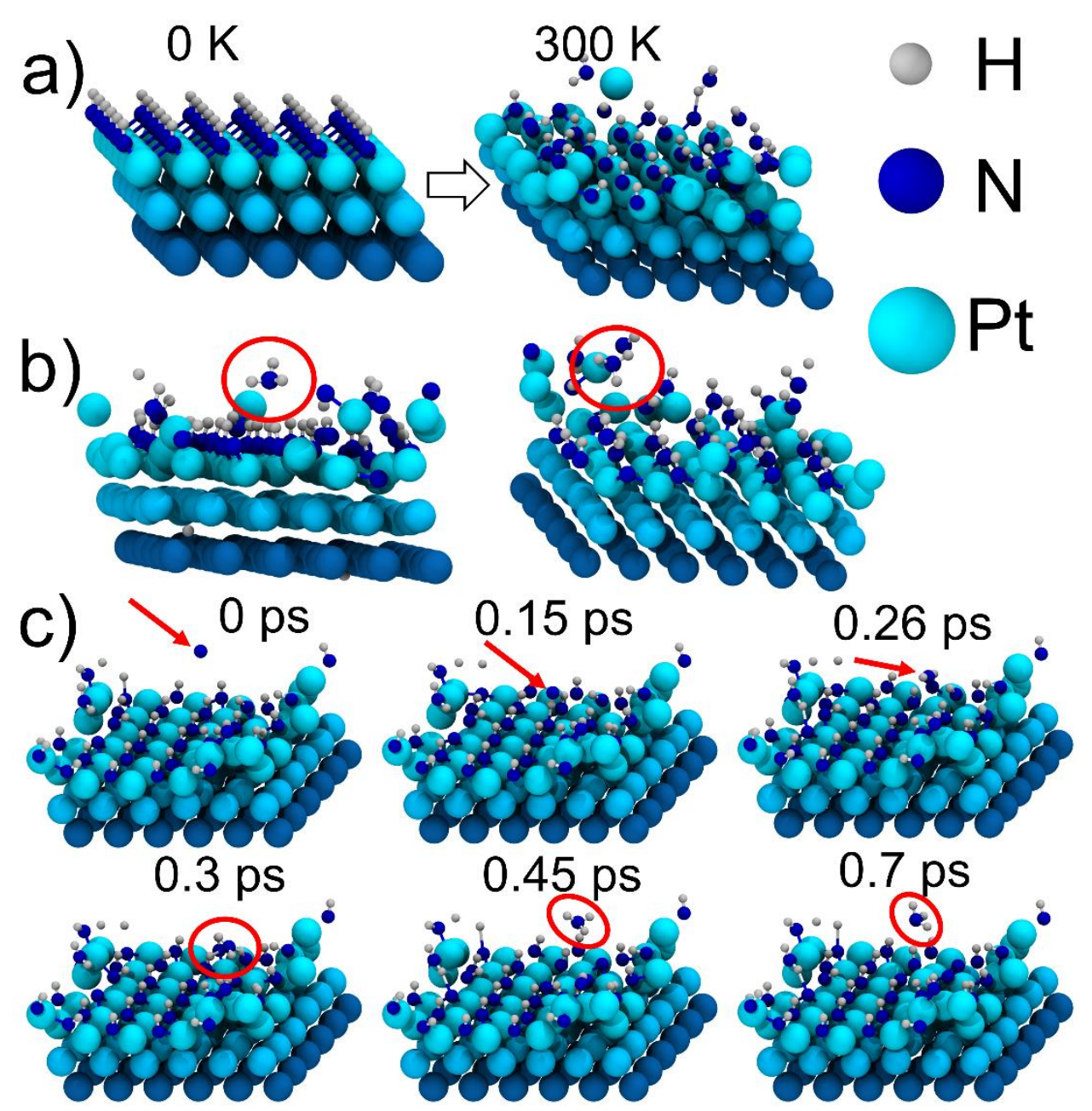

Figure 4. (a) Optimized geometry (left) and configurations after an 8-ps long NVT simulation (right) of an NH-terminated Pt(111) surface. At room temperature, the $\mathrm{NH}$ termination was found to be reasonably stable for a $\mathrm{Pt}$ surface with the desorption of a few $\mathrm{NH}$ and $\mathrm{NH}_{2}$ groups during the simulation. (b) The outcomes of NVE simulations for an atomic nitrogen impinging onto a NH-terminated $\mathrm{Pt}(111)$ surface at $0^{\circ}$ and $30^{\circ}$ angles to the surface normal, with a kinetic energy of $0.1 \mathrm{eV}$. (c) Atomic geometries at various time steps during an NVE simulation of an atomic nitrogen impinging onto a $\mathrm{NH}$-terminated $\mathrm{Pt}(111)$ surface at normal incidence, with a kinetic energy of $0.1 \mathrm{eV}$. For both $H$ and $N$ impingement, we observed the formation and desorption of ammonia on the NH-terminated Pt surface.

impinging atomic nitrogen. For Pt, impinging atomic nitrogen adsorbs onto the surface without the direct abstraction of hydrogen. The saturation of the surface with $\mathrm{NH}$ groups is necessary for activating the direct abstraction mechanism. Interestingly, $\mathrm{NH}_{3}$ formation has been observed for both cases of atomic hydrogen and atomic nitrogen impinging onto the $\mathrm{NH}$-terminated Pt surface. The computational results also explain the similar yield of ammonia that we measure for $\mathrm{Cu}$ and Pt. Our OES measurements strongly suggest that 
the flux of atomic hydrogen to the surface largely exceeds that of atomic nitrogen. Assuming a temperature of $300 \mathrm{~K}$ and using the densities reported earlier $\left(\mathrm{n}_{\mathrm{H}} \sim 4 \times 10^{14} \mathrm{~cm}^{-3}\right.$ and $\left.\mathrm{n}_{\mathrm{N}} \sim 4 \times 10^{13} \mathrm{~cm}^{-3}\right)$, we find that the atomic hydrogen flux to the surface is $\sim 10^{20} \mathrm{~cm}^{-2} \mathrm{~s}^{-1}$ while the atomic nitrogen flux is $\sim 2.7 \times 10^{18} \mathrm{~cm}^{-2} \mathrm{~s}^{-1}$, i.e., the atomic hydrogen flux is $\sim 40$ times larger than that of atomic nitrogen. From the $6 \times 6$ cross-sectional area of the cells used in our BOMD calculations, these fluxes imply that, on average, one hydrogen radical impinges onto that surface every $\sim 350 \mathrm{~ns}$, and one nitrogen radical impinges every $\sim 13 \mu \mathrm{s}$. These timescales are considerably longer than the ones over which we have observed relevant reactions in the BOMD calculations (ps). This result is consistent with a mass-transport limited mechanism in which the formation of ammonia is not limited by surface reaction kinetics (which occur on a ps time scale) but rather by the flux of ammonia precursor to the surface (which occurs on a hundred-ns time scale).

While the difference in ammonia yields between $\mathrm{Cu}$ and $\mathrm{Pt}$ is relatively small, it is reproducible with Pt producing more ammonia than $\mathrm{Cu}$. While our work focuses on the gas-phase dissociation of nitrogen as the dominant activation pathway in low-pressure plasma reactors, this does not rule out that vibrational excitation of molecular nitrogen is also present and contributing to the total ammonia yield. Some dissociative adsorption of nitrogen may occur on metal surfaces even at room temperature, consistent with the proposal from Mehta et al. ${ }^{6}$ This process is likely to be more relevant to Pt compared to $\mathrm{Cu}$ because of the negligible interaction between $\mathrm{Cu}$ and nitrogen $(\mathrm{Cu}$ has a much more positive nitrogen adsorption energy of $+3.4 \mathrm{eV}^{40}$ compared to that of $\mathrm{Pt}$, which is equal to $+0.6 \mathrm{eV}^{6}$ ). This explanation is consistent with the larger ammonia yield that we reproducibly measure for the case of Pt.

To summarize, we have characterized a low-pressure RF-driven plasma reactor for the production of ammonia from a nitrogen-hydrogen mixture. Consistent with previous reports, we observe a weak dependence on catalyst material. Careful characterization suggests that under these conditions, the flux of atomic hydrogen to the catalyst surface largely exceeds that of atomic nitrogen. We have performed largescale BOMD simulations focusing on two metals, $\mathrm{Cu}$ and $\mathrm{Pt}$, which exhibit a significantly different interaction with atomic nitrogen. For the case of $\mathrm{Cu}$, ammonia is formed via direct abstraction of hydrogen from the surface when it is exposed to plasma-produced atomic nitrogen. The case of Pt is more complex. Direct abstraction of ammonia under either atomic nitrogen or hydrogen fluxes can occur rapidly for Pt, but only after the surface is saturated with NH groups. In both scenarios, plasma-produced atomic nitrogen is converted into ammonia on a ps time scale. While our observations are limited to short reaction time scales, which do not capture slower pathways such as Langmuir-Hinshelwood processes, they are consistent with a mass-transport-limited regime and the weak dependence on catalyst material, which we observe and has been reported by several other groups. This work represents the first application of BOMD to the case of plasma catalysis and suggests that this technique is valuable for providing microscopic and mechanistic 
insight into the plasma-surface interaction, with potential future application to other relevant plasmaactivated chemical reactions.

\section{ACKNOWLEDGMENTS}

The authors would like to acknowledge the support of the U.S. Army Research Office under Grant No. W911NF-17-1-0340.

\section{REFERENCES}

(1) Nozaki, T.; Muto, N.; Kado, S.; Okazaki, K., Dissociation of vibrationally excited methane on Ni catalyst: Part 1. Application to methane steam reforming, Catal. Today 2004, 89 (1-2), 57-65.

(2) Nozaki, T.; Muto, N.; Kadio, S.; Okazaki, K., Dissociation of vibrationally excited methane on Ni catalyst: Part 2. Process diagnostics by emission spectroscopy, Catal. Today 2004, 89 (1-2), 67-74.

(3) Kim, J.; Abbott, M. S.; Go, D. B.; Hicks, J. C., Enhancing C-H Bond Activation of Methane via Temperature-Controlled, Catalyst-Plasma Interactions, ACS Energy Letters 2016, 1 (1), 94-99.

(4) Stere, C. E.; Anderson, J. A.; Chansai, S.; Delgado, J. J.; Goguet, A.; Graham, W. G.; Hardacre, C.; Taylor, S. F. R.; Tu, X.; Wang, Z.; Yang, H., Non-Thermal Plasma Activation of Gold-Based Catalysts for Low-Temperature Water-Gas Shift Catalysis, Angewandte Chemie International Edition 2017, 56 (20), 5579-5583.

(5) Xu, S.; Chansai, S.; Stere, C.; Inceesungvorn, B.; Goguet, A.; Wangkawong, K.; Taylor, S. F. R.; Al-Janabi, N.; Hardacre, C.; Martin, P. A.; Fan, X., Sustaining metal-organic frameworks for water-gas shift catalysis by non-thermal plasma, Nature Catalysis 2019, 2 (2), 142-148.

(6) Mehta, P.; Barboun, P.; Herrera, F. A.; Kim, J.; Rumbach, P.; Go, D. B.; Hicks, J. C.; Schneider, W. F., Overcoming ammonia synthesis scaling relations with plasma-enabled catalysis, Nature Catalysis 2018, 1 (4), 269-275.

(7) Mehta, P.; Barboun, P.; Go, D. B.; Hicks, J. C.; Schneider, W. F., Catalysis Enabled by Plasma Activation of Strong Chemical Bonds: A Review, ACS Energy Letters 2019, 4 (5), 1115-1133.

(8) Hong, J.; Aramesh, M.; Shimoni, O.; Seo, D. H.; Yick, S.; Greig, A.; Charles, C.; Prawer, S.; Murphy, A. B., Plasma Catalytic Synthesis of Ammonia Using Functionalized-Carbon Coatings in an Atmospheric-Pressure Non-equilibrium Discharge, Plasma Chem. Plasma Process. 2016, 36 (4), 917-940. (9) Hong, J.; Prawer, S.; Murphy, A. B., Plasma Catalysis as an Alternative Route for Ammonia Production: Status, Mechanisms, and Prospects for Progress, ACS Sustainable Chemistry \& Engineering 2018, 6 (1), 15-31.

(10) Mu, Y.; Xu, S.; Shao, Y.; Chen, H.; Hardacre, C.; Fan, X., Kinetic Study of Nonthermal Plasma Activated Catalytic CO2 Hydrogenation over Ni Supported on Silica Catalyst, Ind. Eng. Chem. Res. 2020.

(11) Xu, S.; Chansai, S.; Shao, Y.; Xu, S.; Wang, Y.-c.; Haigh, S.; Mu, Y.; Jiao, Y.; Stere, C. E.; Chen, H.; Fan, X.; Hardacre, C., Mechanistic study of non-thermal plasma assisted CO2 hydrogenation over Ru supported on $\mathrm{MgAl}$ layered double hydroxide, Applied Catalysis B: Environmental 2020, 268, 118752.

(12) Neyts, E. C.; Bogaerts, A., Understanding plasma catalysis through modelling and simulation-a review, J. Phys. D-Appl. Phys. 2014, 47 (22).

(13) Somers, W.; Bogaerts, A.; van Duin, A. C. T.; Neyts, E. C., Interactions of plasma species on nickel catalysts: A reactive molecular dynamics study on the influence of temperature and surface structure, Applied Catalysis B: Environmental 2014, 154-155, 1-8.

(14) Neyts, E. C.; Ostrikov, K.; Sunkara, M. K.; Bogaerts, A., Plasma Catalysis: Synergistic Effects at the Nanoscale, Chemical Reviews 2015, 115 (24), 13408-13446.

(15) Whitehead, J. C., Plasma-catalysis: the known knowns, the known unknowns and the unknown unknowns, Journal of Physics D: Applied Physics 2016, 49 (24), 243001. 
(16) Galloway, J. N., The global nitrogen cycle: changes and consequences, Environmental Pollution 1998, 102 (1, Supplement 1), 15-24.

(17) Baltrusaitis, J. Sustainable Ammonia Production; American Chemical Society: 2017/11/06, 2017; pp 9527-9527.

(18) Cherkasov, N.; Ibhadon, A. O.; Fitzpatrick, P., A review of the existing and alternative methods for greener nitrogen fixation, Chemical Engineering and Processing: Process Intensification 2015, 90, $24-$ 33.

(19) Schrock, R. R., Reduction of dinitrogen, Proceedings of the National Academy of Sciences 2006, 103 (46), 17087-17087.

(20) Barboun, P.; Mehta, P.; Herrera, F. A.; Go, D. B.; Schneider, W. F.; Hicks, J. C., Distinguishing Plasma Contributions to Catalyst Performance in Plasma-Assisted Ammonia Synthesis, ACS Sustainable Chemistry \& Engineering 2019, 7 (9), 8621-8630.

(21) Shah, J.; Wang, W.; Bogaerts, A.; Carreon, M. L., Ammonia Synthesis by Radio Frequency Plasma Catalysis: Revealing the Underlying Mechanisms, ACS Applied Energy Materials 2018, 1 (9), 4824-4839.

(22) Ben Yaala, M.; Saeedi, A.; Scherrer, D.-F.; Moser, L.; Steiner, R.; Zutter, M.; Oberkofler, M.; De Temmerman, G.; Marot, L.; Meyer, E., Plasma-assisted catalytic formation of ammonia in N2-H2 plasma on a tungsten surface, Phys. Chem. Chem. Phys. 2019, 21 (30), 16623-16633.

(23) Iwamoto, M.; Akiyama, M.; Aihara, K.; Deguchi, T., Ammonia Synthesis on Wool-Like Au, Pt, $\mathrm{Pd}, \mathrm{Ag}$, or $\mathrm{Cu}$ Electrode Catalysts in Nonthermal Atmospheric-Pressure Plasma of $\mathrm{N} 2$ and $\mathrm{H} 2, A C S$ Catalysis 2017, 7 (10), 6924-6929.

(24) Helden, J. H. v.; Wagemans, W.; Yagci, G.; Zijlmans, R. A. B.; Schram, D. C.; Engeln, R.; Lombardi, G.; Stancu, G. D.; Röpcke, J., Detailed study of the plasma-activated catalytic generation of ammonia in N2-H2 plasmas, J. Appl. Phys. 2007, 101 (4), 043305.

(25) van 't Veer, K.; Reniers, F.; Bogaerts, A., Zero-dimensional modeling of unpacked and packed bed dielectric barrier discharges: the role of vibrational kinetics in ammonia synthesis, Plasma Sources Science and Technology 2020, 29 (4), 045020.

(26) Carrasco, E.; Jiménez-Redondo, M.; Tanarro, I.; Herrero, V. J., Neutral and ion chemistry in low pressure dc plasmas of $\mathrm{H} 2 / \mathrm{N} 2$ mixtures: routes for the efficient production of $\mathrm{NH} 3$ and NH4+, Phys. Chem. Chem. Phys. 2011, 13 (43), 19561-19572.

(27) Yamijala, S. S. R. K. C.; Ali, Z. A.; Wong, B. M., Acceleration vs Accuracy: Influence of Basis Set Quality on the Mechanism and Dynamics Predicted by Ab Initio Molecular Dynamics, The Journal of Physical Chemistry C 2019, 123 (41), 25113-25120.

(28) Tsona, N. T.; Bork, N.; Loukonen, V.; Vehkamäki, H., A Closure Study of the Reaction between Sulfur Dioxide and the Sulfate Radical Ion from First-Principles Molecular Dynamics Simulations, The Journal of Physical Chemistry A 2016, 120 (7), 1046-1050.

(29) Lin, W.; Stocker, K. M.; Schatz, G. C., Mechanisms of Hydrogen-Assisted CO2 Reduction on Nickel, J. Am. Chem. Soc. 2017, 139 (13), 4663-4666.

(30) Coburn, J. W.; Chen, M., Optical emission spectroscopy of reactive plasmas: a method for correlating emission intensities to reactive particle density, J. Appl. Phys. 1980, 51 (6), 3134-3136.

(31) Tatarova, E.; Dias, F. M.; Gordiets, B.; Ferreira, C. M., Molecular dissociation in N2-H2 microwave discharges, Plasma Sources Science and Technology 2004, 14 (1), 19-31.

(32) database, https://www.nist.gov/pml/atomic-spectra-database

(33) database, www.lxcat.net

(34) Hagelaar, G. J. M.; Pitchford, L. C., Solving the Boltzmann equation to obtain electron transport coefficients and rate coefficients for fluid models, Plasma Sources Science and Technology 2005, 14 (4), 722.

(35) Version 12/2017 available at http://www.bolsig.laplace.univ-tlse.fr/index.html

(36) Amorim, J.; Baravia, G.; Ricard, A., Production of N, H, and NH active species in N2-H2 dc flowing discharges, Plasma Chem. Plasma Process. 1995, 15 (4), 721-731. 
(37) Gordiets, B.; Ferreira, C. M.; Pinheiro, M. J.; Ricard, A., Self-consistent kinetic model of lowpressure - flowing discharges: I. Volume processes, Plasma Sources Science and Technology 1998, 7 (3), 363-378.

(38) Gordiets, B.; Ferreira, C. M.; Pinheiro, M. J.; Ricard, A., Self-consistent kinetic model of lowpressure - flowing discharges: II. Surface processes and densities of N, H, species, Plasma Sources Science and Technology 1998, 7 (3), 379-388.

(39) Ricard, A.; Henriques, J.; Cousty, S.; Villeger, S.; Amorim, J., Determination of N-, H- and OAtom Densities in N2-H2 and in N2-O2 Gas Mixtures by Optical Actinometry in Flowing Microwave Discharges and by NO Titration in Post-Discharges, Plasma Processes and Polymers 2007, 4 (S1), S965S968.

(40) Pang, X.-Y.; Xue, L.-Q.; Wang, G.-C., Adsorption of Atoms on Cu Surfaces: A Density Functional Theory Study, Langmuir 2007, 23 (9), 4910-4917. 